\title{
Soll man erneut antikoagulieren?
}

Fragestellung: Sollten Patienten mit Vorhofflimmern (VHF), die unter oraler Antikoagulation eine intrakranielle Blutung erlitten haben, zu einem späteren Zeitpunkt erneut antikoaguliert werden?

Hintergrund: Bei Patienten mit VHF reduziert die orale Antikoagulation massiv das Schlaganfallrisiko. Die gefürchtetste Komplikation insbesondere mit Vitamin-K-Antagonisten (VKA) sind intrakranielle Blutungen. Sie haben eine Sterblichkeit von bis zu $40 \%$. Ein noch weitgehend ungelöstes Problem ist die Frage, ob bei Patienten mit VHF, die unter oraler Antikoagulation eine intrakranielle Blutung erlitten haben, zu einen späteren Zeitpunkt erneut antikoaguliert werden sollte.

Patienten und Methodik: Im dänischen Patientenregister wurden Patienten identifiziert, die zwischen 1997 und 2013 aufgrund von VHF mit oralen Antikoagulanzien behandelt wurden und unter dieser Therapie eine intrakranielle Blutung erlitten. Ab sechs Wochen nach der intrakraniellen Blutung wurden ischämische Insulte, die Gesamtsterblichkeit, erneute intrakranielle Blutungen und schwerwiegende Blutungskomplikationen erfasst. Die Patienten wurden in drei Gruppen eingeteilt: keine Behandlung, erneute oral Antikoagulation oder Therapie mit Thrombozytenfunktionshemmern.

Nielsen PB, Larsen TB, Skjøth F et al. Restarting anticoagulant treatment after intracranial haemorrhage in patients with atrial fibrillation and the impact on recurrent stroke, mortality and bleeding: A nation-wide cohort study. Circulation 2015; 132: 517-25
Ergebnisse: Insgesamt wurden 6.138 Patienten mit VHF identifiziert, bei denen eine intrakranielle Blutung aufgetreten war. In den ersten sechs Wochen nach dem Ereignis verstarben 1.652 Patienten. 1.752 Patienten waren in den sechs Monaten vor der intrakraniellen Blutung mit oralen Antikoagulanzien behandelt worden. $65 \%$ der Patienten hatten zuvor ausschließlich VKA erhalten und $33 \%$ eine Kombinationstherapie aus VKA und Thrombozytenfunktionshemmern. $2 \%$ der Patienten hatten neue Antikoagulanzien (NOAK) erhalten. Innerhalb der Patientenpopulation hatten 812 eine intrazerebrale Blutung, 755 ein subdurales Hämatom und 185 eine Subarachnoidalblutung erlitten.

Die Ereignisrate pro 100 Patientenjahre betrug für den kombinierten Endpunkt ischämischer Insult und Sterblichkeit bei den unbehandelten Patienten 27,3 und bei den erneut antikoagulierten Patienten 13,6 (Hazard Ratio [HR] 0,55). Patienten, die mit Plättchenhemmern behandelt wurden, hatten eine Ereignisrate von 25,7 (HR 0,87, nicht signifikant).

Die Ereignisraten für ischämischen Schlaganfall betrugen unter oraler Antikoagulation 5,3 pro Jahr und ohne Behandlung 10,4 pro Jahr (HR 0,59, knapp nicht signifikant). Unter Plättchenhemmertherapie lag die Ereignisrate bei 10,3 (HR 0,98). Die Sterblichkeitsraten betrugen 9,7 für orale Antikoagulanzien versus 19,1, für keine antithrombotische Behandlung (Odds Ratio 0,55) und 19,5 für Thrombozytenfunktionshemmer (HR 0,90).

Die Raten schwerwiegender Blutungskomplikationen waren in den drei Patientengruppen vergleichbar. Die Rate erneuter intrakranieller Blutungen pro 100 Patientenjahre betrug 8,6 für mit Antikoagulanzien behandelte Patienten, 8,0 für unbehandelte $\mathrm{Pa}$ tienten und 5,3 für mit Plättchenhemmern behandelte Patienten. Die Unterschiede waren statistisch nicht signifikant.

Schlussfolgerungen: Bei Patienten, die unter Antikoagulanzien zur Schlaganfallprävention bei VHF eine intrakranielle Blutung erleiden, reduziert eine erneute Antikoagulation zu einem späteren Zeitpunkt signifikant das Risiko von Schlaganfall und Tod. Thrombozytenfunktionshemmer sind entweder unwirksam oder deutlich weniger wirksam. Die Rate erneuter intrakranieller Blutungen und schwerwiegender anderer Blutungskomplikationen ist bei Patienten, die erneut antikoaguliert werden, nicht erhöht.

\section{- Kommentar von Hans-Christoph Diener, Essen}

\section{Nur sehr wenige Patienten in dieser Studie erhielten NOAK}

Diese große populationsbezogene Studie belegt ebenso wie die vor kurzem aus Erlangen publizierte Studie [1], dass offenbar Patienten mit Vorhofflimmern, die eine intrakranielle Blutung erlitten haben, weiterhin ein hohes Schlaganfallrisiko haben. Für diese Patientenpopulation ist eine erneute orale Antikoagulation wirksamer als eine Behandlung mit Thrombozytenfunktionshemmern und erhöht nicht das Risiko intra- oder extrakranieller schwerwiegender Blutungskomplikationen.

Die Studie hat auch eine Reihe von Problemen und ungelösten Fragen: So hängt die Entscheidung eines Klinikers, einen Patienten erneut zu antikoagulieren, sicher davon ab, wie hoch er das Risiko für einen erneuten ischämischen Insult oder eine erneute Blutung einschätzt. In der Datenbank sind keine INR-Werte verzeichnet, sodass sich nicht ermessen lässt, bei wie vielen Patienten die intrakranielle Blutung durch eine fehlerhafte Antikoagulation bedingt war.

Letztendlich kann die wichtige Frage ob, und insbesondere wann, in dieser Patientengruppe eine erneute Antikoagulation durchgeführt werden sollte, nur durch eine prospektive Studie beantwortet werden. Zudem war die Zahl der Patienten, die neue Antikoagulanzien erhielten, die mit einem deutlich verringerten Risiko für intrakranielle Blutungen einhergehen, verschwindend gering.

\footnotetext{
Referenz:

1. Kuramatsu JB et al. JAMA 2015; 313: 824-36
} 